\title{
Acceptance and Opinions of Intanza/IDflu Intradermal Influenza Vaccine in the Czech Republic and Turkey
}

Roman Prymula · Gaye Usluer · Serdar Altinel · Radka Sichova · Françoise Weber

To view enhanced content go to www.advancesintherapy.com

Received: November 10, 2011 / Published online: January 3, 2012

(c) The Author(s) 2012. This article is published with open access at Springerlink.com

\section{ABSTRACT}

Introduction: Intanza ${ }^{\circledR} / \mathrm{IDflu}^{\circledR}$ (Sanofi Pasteur SA, Lyon, France), a split-virion, trivalent influenza vaccine delivered by intradermal injection with a microinjection system, became available in adults 18 -59 years of age $(9 \mu \mathrm{g})$ and $\geq 60$ years of age $(15 \mu \mathrm{g})$ as of the 2010/2011 northern hemisphere influenza season. Methods: This study assessed the acceptability of intradermal vaccination

Roman Prymula

University Hospital, Hradec Králové, Czech Republic

Gaye Usluer

Osmangazi University Hospital, Eskisehir, Turkey

Serdar Altinel

Sanofi Pasteur, Istanbul, Turkey

Radka Sichova

Sanofi Pasteur, Prague, Czech Republic

Françoise Weber $(\bowtie)$

Sanofi Pasteur, 2 avenue Pont Pasteur, 69007 Lyon,

France. Email: francoise.weber@sanofipasteur.com

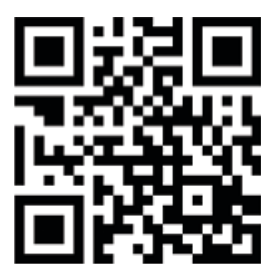

Enhanced content for Advances in Therapy articles is available on the journal web site: www.advancesintherapy.com with Intanza/IDflu in routine clinical practice in adult vaccinees and their vaccine prescribers. Vaccine prescribers and adults who had elected to be vaccinated with Intanza/IDflu during the 2010/2011 northern hemisphere influenza season were recruited to complete surveys about their opinions of influenza vaccination and their acceptance of the intradermal vaccination. Czech subjects 18-59 years of age were vaccinated with the $9 \mu \mathrm{g}$ formulation and those $\geq 60$ years of age with the $15 \mu \mathrm{g}$ formulation of Intanza/IDflu. All Turkish subjects were vaccinated with the $9 \mu \mathrm{g}$ formulation, as Intanza/IDflu $15 \mu \mathrm{g}$ was not available in Turkey at the time the survey was conducted. Results: One thousand and twelve vaccinees and 28 vaccine prescribers in the Czech Republic, and 249 vaccinees and 15 vaccine prescribers in Turkey completed questionnaires. Overall, $96.1 \%$ of vaccinees were satisfied or very satisfied with Intanza/IDflu. The main reason for satisfaction was that the injection was considered minimally painful. Most (93.9\%) vaccinees reported that they would prefer to receive the same vaccination next year. Furthermore, 95.3\% of vaccine prescribers were satisfied or very satisfied with the intradermal vaccine, and $82.6 \%$ preferred intradermal over intramuscular vaccination. Conclusions: Intradermal vaccination for seasonal 
influenza using Intanza/IDflu is well accepted by adult vaccinees and vaccine prescribers. By providing an additional, well-accepted method, Intanza/IDflu might help increase seasonal influenza vaccination rates in adults.

Keywords: acceptability; influenza; Intanza/ IDflu; intradermal; seasonal; trivalentinactivated; vaccine; vaccinee satisfaction

\section{INTRODUCTION}

Seasonal influenza is a threat to public health with a major socioeconomic impact. ${ }^{1}$ Worldwide, influenza is responsible for 3-5 million cases of severe illness and 250,000500,000 deaths each year, ${ }^{2}$ most of which are in high-risk groups, including the elderly ( $\geq 65$ years), children up to 5 years of age, pregnant women, and people with certain chronic diseases and conditions. ${ }^{2-6}$ Workingage adults are at lower risk of complications, hospitalization, and death than high-risk groups, but they account for approximately one-third of the annual cost of seasonal influenza, mostly due to work absenteeism and reduced productivity.,8

Vaccination is the most cost-effective medical intervention against seasonal influenza.9,10 The World Health Organization currently recommends that by $2014 / 15$, influenza vaccination coverage should reach $75 \%$ in elderly adults and all persons with underlying diseases, ${ }^{11}$ targets officially adopted in 2009 by the European Union. ${ }^{12}$ In the US, universal influenza vaccination has been recommended for all children $\geq 6$ months of age since 2010 . $^{13}$ The influenza vaccine coverage, however, remains far below these targets. In European countries, influenza vaccine coverage during the 2006-2007 influenza season ranged from $2 \%-82 \%$ in adults over 65 years of age and from $28 \%-75 \%$ in clinical risk groups. ${ }^{14}$
In the US, the most recently reported seasonal influenza coverage rates were $28 \%$ in adults 18-49 years of age not at risk, 36\% in adults 18-49 years of age at high risk (ie, with underlying conditions), $45 \%$ in adults 50-64 years of age, and $68 \%$ in adults 65 years of age and older. ${ }^{15}$

Seasonal influenza vaccines have been generally administered by intramuscular (i.m.) injection. Vaccination by the intradermal (i.d.) route using a microinjection system has been proposed as a way of improving influenza vaccine uptake because it uses a needle 10 times shorter than the i.m. needle, and because it allows rapid and safe vaccination. ${ }^{16}$ Intanza $^{\circledR} /$ IDflu $^{\circledR}$ (Sanofi Pasteur SA, Lyon, France), the first microneedle, trivalent, inactivated influenza vaccine, is administered using the Soluvia ${ }^{\mathrm{TM}}$ microinjection system (Becton Dickinson, Franklin Lakes, NJ, USA), which consists of a prefilled $0.5 \mathrm{~mL}$ glass syringe fitted with a 30-gauge, short-bevel microneedle that protrudes $1.5 \mathrm{~mm}$ from a depth-limiting tip. ${ }^{17}$ The microinjection system also includes a shield that covers the needle after use, preventing needle reuse and accidental needle-stick injuries. Intanza/IDflu was approved in Europe in 2009 by the European Medicines Agency for the prevention of influenza in both working-age adults (18-59 years of age; $9 \mu$ g hemagglutinin per strain) and elderly adults ( $\geq 60$ years of age; $15 \mu$ g hemagglutinin per strain). ${ }^{17,18}$ Clinical studies have shown that the $9 \mu \mathrm{g}$ formulation of Intanza/IDflu has noninferior immunogenicity and that the $15 \mu \mathrm{g}$ formulation has greater immunogenicity than Vaxigrip ${ }^{\circledR}$ (Sanofi Pasteur SA, Lyon, France), an i.m., split-virion, trivalent influenza vaccine that has been used for more than 45 years and has an established record of safety and efficacy. ${ }^{19-23}$ In addition, the systemic safety profile of both formulations of Intanza/ IDflu are similar to that of Vaxigrip. Local reactions are more common with Intanza/IDflu 
than Vaxigrip, which is as expected because the injection site reactions occur in the skin rather than the muscle, where local reactions can be more easily observed.

Recommendations in Turkey are for influenza vaccination in people 65 years of age and older living in nursing homes or elderly care centers; in all patients with chronic pulmonary, cardiac, metabolic, or kidney diseases, hemoglobinopathies, immune deficiency, or receiving immunosuppressants; and in adolescents and children receiving chronic aminosalicylates. ${ }^{24}$ In the Czech Republic, recommendations are for influenza vaccination in people with chronic pulmonary disease, cardiovascular disease (except hypertension), or renal disease. ${ }^{25}$ In the current study, the authors assessed the acceptability of i.d. influenza vaccination with Intanza/IDflu in routine clinical use during the 2010/2011 northern hemisphere influenza season in Turkey and the Czech Republic, and examined vaccinee attitudes towards vaccination for seasonal influenza.

\section{MATERIALS AND METHODS}

\section{Study Design and Participants}

This was an uncontrolled, noninterventional, observational, multicenter study on the acceptance of Intanza/IDflu in routine clinical practice by adult subjects and healthcare practitioners. The survey was carried out in the Czech Republic between October 6 and November 10, 2010 and in Turkey between December 31, 2010 and January 31, 2011. The study was performed in accordance with local laws, rules, and regulations, including the Declaration of Helsinki, the guidelines of Good Pharmacoepidemiology Practices (Appendix 5), European Directive for Data Protection (95/46/EC), Volume 9A, and national pharmacovigilance regulations. Healthcare professionals at general practice and occupational health clinics in Turkey and the Czech Republic conducted the surveys. In the Czech Republic, an offer to participate in the study was extended to the Ministry of Defense, with most of the military public health physicians taking part. In addition, the study was offered to the Ministry of the Interior and to the Society of General Practitioners from which regional clinics participated. In Turkey, participating physicians were selected by Sanofi Pasteur through collaboration with a contract research organization. Selection of participating physicians was made on the basis of their potential patient populations. The participating clinics consecutively enrolled adults $>18$ years old (Czech Republic) or adults 18-59 years old (Turkey) to be vaccinated with Intanza/IDflu and who were willing to complete surveys. No restrictions were made with respect to the urbanization, sex ratio, socioeconomic status, presence of chronic diseases, or other demographic factors. Each vaccinee was required to provide written informed consent to receive the vaccine and had to be vaccinated to be included in the survey. Exclusion criteria included hypersensitivity to the active substances or any of the excipients. Immunization was postponed in vaccinees with febrile illness or acute infection.

\section{Treatments and Assessments}

In the Czech Republic, subjects $18-59$ years of age were vaccinated with Intanza/IDflu $9 \mu \mathrm{g}$ and those $\geq 60$ years of age were vaccinated with Intanza/IDflu $15 \mu \mathrm{g}$. All subjects in Turkey were vaccinated with Intanza/IDflu $9 \mu$. Immediately after vaccination, vaccinees completed a self-administered questionnaire that collected demographic information and 
asked the subjects about their perception of the risk of getting influenza, their influenza vaccination history, their satisfaction with the i.d. vaccination, and their vaccine preference for the next year. Vaccinees were also contacted by telephone 8 days after vaccination and asked again about their vaccine preference for next year. At the end of the study, prescribers of the vaccine completed a questionnaire collecting demographic information and asking them about their satisfaction with Intanza/IDflu.

\section{Statistical Analysis}

All analyses were performed using SAS version 9.1 (SAS Institute, Cary, NC, USA). Categorical variables were described by the percentage of each response choice, with missing data excluded in the calculation of percentage.

\section{RESULTS}

\section{Intanza/IDflu Vaccinee Responses}

A total of 1261 vaccinees completed the survey (Table 1). In the Czech Republic, the majority of vaccinees were men, whereas in Turkey, the majority of vaccinees were women. All 1012 vaccinees in the Czech Republic were vaccinated according to the protocol, whereas 14 of 249 in Turkey were above the age for enrollment (ie, $\geq 60$ years of age, which constituted off-label use of Intanza/IDflu $9 \mu \mathrm{g}$ ) and, therefore, were not vaccinated according to the protocol.

\section{Risk Perception for Contracting Influenza}

Most vaccinees in both countries felt at risk of contracting influenza. In the Czech Republic, $66.7 \%$ of adults $18-59$ years of age and $89.2 \%$ of elderly adults felt at risk, whilst in Turkey, 90.3\% felt at risk (Table 2). In Turkish and Czech adults 18-59 years of age, the most frequent reason for feeling at risk was "I come into contact with many people," whereas in elderly Czech adults, the most common reason was "I have a chronic illness" followed by "I am at risk because of my elderly age."

Only $10.8 \%$ of elderly vaccinees in the Czech Republic, $33.3 \%$ of Czech adults $18-59$ years of age, and $9.7 \%$ of vaccinees in Turkey did not feel at risk of contracting influenza. For Turkish vaccinees and Czech vaccinees $18-59$ years of age, the most common reason for not feeling at risk was "I rely on my natural defenses/ immunity system." In Czech adults $\geq 60$ years of age, the most common reason was "I have no chronic illness that puts me at risk of the flu."

\section{Frequency of Vaccination}

In the Czech Republic, most vaccinees reported being vaccinated for influenza every year (65.4\% of adults $18-59$ years of age and $79.6 \%$ of adults $\geq 60$ years of age). Less than $20 \%$ reported that they had not been previously vaccinated (19.3\% of adults $18-59$ years of age and $12.6 \%$ of adults $\geq 60$ years of age). Of those previously vaccinated, $83.1 \%$ of adults $18-59$ years of age and $93.1 \%$ of adults $\geq 60$ years of age had been vaccinated the year before. In contrast, more than half of vaccinees in Turkey (51.6\%) reported that they had not been previously vaccinated for seasonal influenza (Table 2). In addition, just over half of those previously vaccinated $(57.1 \%)$ had been vaccinated the year before.

\section{Main Reasons for Being Vaccinated}

In both Turkey and the Czech Republic, the most common reason prompting vaccination, cited by more than half of the vaccinees, was the advice of a physician (Table 2). In Czech vaccinees, the second-most common reason was "my own belief in the importance of flu 
Table 1. Demographics.

\begin{tabular}{|c|c|c|c|c|c|c|}
\hline & \multicolumn{4}{|c|}{ Czech Republic } & \multirow{2}{*}{\multicolumn{2}{|c|}{$\frac{\text { Turkey }}{\text { Intanza/IDflu } 9 \mu \mathrm{g}}$}} \\
\hline & \multicolumn{2}{|c|}{ Intanza/IDflu $9 \mu \mathrm{g}$} & \multicolumn{2}{|c|}{ Intanza/IDflu $15 \mu \mathrm{g}$} & & \\
\hline & $n$ & $\%$ & $n$ & $\%$ & $n$ & $\%$ \\
\hline Age (years) & \multicolumn{2}{|c|}{$n=845$} & \multicolumn{2}{|c|}{$n=167$} & \multicolumn{2}{|c|}{$n=249$} \\
\hline $18-49$ & 693 & 82.0 & 0 & 0.0 & 144 & 57.8 \\
\hline $50-59$ & 152 & 18.0 & 0 & 0.0 & 91 & 36.5 \\
\hline $60-74$ & 0 & 0.0 & 120 & 71.9 & $9^{*}$ & 3.6 \\
\hline$\geq 75$ & 0 & 0.0 & 47 & 28.1 & $5^{*}$ & 2.0 \\
\hline Sex & \multicolumn{2}{|c|}{$n=845$} & \multicolumn{2}{|c|}{$n=165$} & \multicolumn{2}{|c|}{$n=249$} \\
\hline Male & 677 & 80.1 & 88 & 53.3 & 102 & 41.0 \\
\hline Female & 168 & 19.9 & 77 & 46.7 & 147 & 59.0 \\
\hline
\end{tabular}

*Off-label use.

vaccination" (41.7\% of adults $18-59$ years of age and $31.1 \%$ of adults $\geq 60$ years of age), but this reason was cited by only $6.6 \%$ of Turkish vaccinees. Instead, the advice of a physician's office assistant or nurse was the second-most common prompting vaccination in Turkey.

\section{Main Reasons for Missing Vaccination}

In Turkey, the most common reason for missing previous vaccinations was "I was not encouraged to be vaccinated" (Table 2), whereas in the Czech Republic, not being encouraged to be vaccinated was reported as the reason for missing vaccinations by only $3.8 \%$ of adults 18 -59 years of age and only $23.3 \%$ of adults $\geq 60$ years of age. Instead, the most common reason for missing previous vaccinations was "I did not feel that I was at risk of catching the flu."

\section{Vaccinees' Opinions of the Most Effective Reminder to be Vaccinated}

In both countries, more than two-thirds of vaccinees indicated that advice of their physician or general practitioner (GP) would be the most effective reminder to be vaccinated (Table 2). A postcard, email, or text message sent by the nurse, physician's assistant, or physician's/GP's clinic was cited as the second most effective reminder.

\section{Vaccinee Satisfaction with Intanza/IDflu}

Overall, 96.1\% of vaccinees reported being satisfied or very satisfied with Intanza/IDflu (Table 2). This included $96.0 \%$ of vaccinees in Turkey, and in the Czech Republic, 95.6\% of vaccinees $18-59$ years of age and $98.8 \%$ of vaccinees $\geq 60$ years of age. Of respondents vaccinated every year for influenza (and, therefore, previously vaccinated i.m.), 90.9\% $(60 / 66)$ in Turkey and $98.0 \%(672 / 686)$ in the Czech Republic were satisfied or very satisfied. Of those not previously vaccinated, $96.8 \%$ $(122 / 126)$ in Turkey and 93.5\% (172/184) in the Czech Republic were satisfied or very satisfied. The main reason for satisfaction in both countries was "the injection was minimally painful/only hurt a little," followed by "the vaccination/administration process was quick." According to multivariate statistical analysis, in both countries, satisfaction with Intanza/IDflu 
Table 2. Vaccinee responses (continued on next page).

\begin{tabular}{|c|c|c|c|c|c|c|}
\hline & & & \multicolumn{4}{|c|}{ Czech Republic } \\
\hline & \multicolumn{2}{|c|}{ Turkey } & \multicolumn{2}{|c|}{$(18-59$ years $)$} & \multicolumn{2}{|c|}{$(\geq 60$ years $)$} \\
\hline & \multirow{2}{*}{\multicolumn{2}{|c|}{$\begin{array}{c}\text { Intanza/IDflu } \\
9 \mu \mathrm{g}\end{array}$}} & \multirow{2}{*}{\multicolumn{2}{|c|}{$\begin{array}{c}\text { Intanza/IDflu } \\
9 \mu \mathrm{g}\end{array}$}} & \multirow{2}{*}{\multicolumn{2}{|c|}{$\begin{array}{c}\text { Intanza/IDflu } \\
15 \mu \mathrm{g}\end{array}$}} \\
\hline & & & & & & \\
\hline & $n$ & $\%$ & $n$ & $\%$ & $n$ & $\%$ \\
\hline Do you feel at risk of catching the flu? & \multicolumn{2}{|c|}{$n=248$} & \multicolumn{2}{|c|}{$n=845$} & \multicolumn{2}{|c|}{$n=167$} \\
\hline Yes & 224 & 90.3 & 564 & 66.7 & 149 & 89.2 \\
\hline No & 24 & 9.7 & 281 & 33.3 & 18 & 10.8 \\
\hline Reason for feeling at risk & \multicolumn{2}{|c|}{$n=216$} & \multicolumn{2}{|c|}{$n=563$} & \multicolumn{2}{|c|}{$n=148$} \\
\hline I have a chronic illness & 34 & 15.7 & 49 & 8.7 & 70 & 47.3 \\
\hline I am at risk because of my elderly age & 11 & 5.1 & 8 & 1.4 & 45 & 30.4 \\
\hline I come into contact with many people & 146 & 67.6 & 443 & 78.7 & 27 & 18.2 \\
\hline I previously had the flu & 25 & 11.6 & 63 & 11.2 & 6 & 4.1 \\
\hline Reason for not feeling at risk & \multicolumn{2}{|c|}{$n=22$} & \multicolumn{2}{|c|}{$n=281$} & \multicolumn{2}{|c|}{$n=17$} \\
\hline I have no chronic illness that puts me at risk of the flu & 5 & 22.7 & 88 & 31.3 & 8 & 47.1 \\
\hline I am too young to be at risk & 3 & 13.6 & 15 & 5.3 & 0 & 0.0 \\
\hline I try to avoid crowded places/environments & 5 & 22.7 & 10 & 3.6 & 4 & 23.5 \\
\hline I rely on my natural defenses/immunity system & 7 & 31.8 & 119 & 42.3 & 3 & 17.6 \\
\hline I have a healthy lifestyle & 2 & 9.1 & 49 & 17.4 & 2 & 11.8 \\
\hline Who/what prompted you to receive your flu vaccination today? & \multicolumn{2}{|c|}{$n=243$} & \multicolumn{2}{|c|}{$n=845$} & \multicolumn{2}{|c|}{$n=167$} \\
\hline Advice of physician or GP & 160 & 65.8 & 425 & 50.3 & 96 & 57.5 \\
\hline Advice of physician's office assistant or nurse & 51 & 21.0 & 11 & 1.3 & 7 & 4.2 \\
\hline Advice of pharmacist & 0 & 0.0 & 6 & 0.7 & 0 & 0.0 \\
\hline Advice of family, friend, or colleague & 16 & 6.6 & 48 & 5.7 & 10 & 6.0 \\
\hline My own belief in the importance of flu vaccination & 16 & 6.6 & 352 & 41.7 & 52 & 31.1 \\
\hline Poster or communication in the waiting room or pharmacy & 0 & 0.0 & 3 & 0.4 & 2 & 1.2 \\
\hline How often do you receive a flu vaccine? & \multicolumn{2}{|c|}{$n=244$} & \multicolumn{2}{|c|}{$n=844$} & \multicolumn{2}{|c|}{$n=167$} \\
\hline Every year & 66 & 27.0 & 552 & 65.4 & 133 & 79.6 \\
\hline Every 2 years & 22 & 9.0 & 47 & 5.6 & 4 & 2.4 \\
\hline Less than every 2 years & 30 & 12.3 & 82 & 9.7 & 9 & 5.4 \\
\hline Have not in the past - this is my first time & 126 & 51.6 & 163 & 19.3 & 21 & 12.6 \\
\hline When did you last receive a flu vaccine? & \multicolumn{2}{|c|}{$n=119$} & \multicolumn{2}{|c|}{$n=681$} & \multicolumn{2}{|c|}{$n=145$} \\
\hline Last year & 68 & 57.1 & 566 & 83.1 & 135 & 93.1 \\
\hline Two years ago & 22 & 18.5 & 55 & 8.1 & 5 & 3.4 \\
\hline Several years ago & 15 & 12.6 & 50 & 7.3 & 3 & 2.1 \\
\hline I don't remember & 14 & 11.8 & 10 & 1.5 & 2 & 1.4 \\
\hline
\end{tabular}


Table 2 (continued). Vaccinee responses.

\begin{tabular}{|c|c|c|c|c|c|c|}
\hline Main reason for missing flu vaccination last year or the year before & \multicolumn{2}{|c|}{$n=176$} & \multicolumn{2}{|c|}{$n=289$} & \multicolumn{2}{|c|}{$n=30$} \\
\hline I was not encouraged to be vaccinated & 97 & 55.1 & 11 & 3.8 & 7 & 23.3 \\
\hline I have a fear of injections or needles & 20 & 11.4 & 9 & 3.1 & 5 & 16.7 \\
\hline I was afraid of side effects or contracting flu from the vaccine & 27 & 15.3 & 58 & 20.1 & 4 & 13.3 \\
\hline I did not feel that I was at risk of catching the flu & 32 & 18.2 & 211 & 73.0 & 14 & 46.7 \\
\hline $\begin{array}{l}\text { Which of the following would be most effective in reminding } \\
\text { you of future flu vaccination? }\end{array}$ & \multicolumn{2}{|c|}{$n=243$} & \multicolumn{2}{|c|}{$n=845$} & \multicolumn{2}{|c|}{$n=167$} \\
\hline Advice from physician or GP & 192 & 79.0 & 546 & 64.6 & 114 & 68.3 \\
\hline $\begin{array}{l}\text { Postcard, email, SMS sent by the nurse, physician's assistant, } \\
\text { or physician's/GP's clinic }\end{array}$ & 30 & 12.3 & 117 & 13.8 & 23 & 13.8 \\
\hline Reminder from a pharmacist & 1 & 0.4 & 7 & 0.8 & 1 & 0.6 \\
\hline Advice or reminder from family or a friend & 7 & 2.9 & 42 & 5.0 & 7 & 4.2 \\
\hline Articles in the media & 7 & 2.9 & 37 & 4.4 & 15 & 9.0 \\
\hline None & 6 & 2.5 & 96 & 11.4 & 7 & 4.2 \\
\hline How satisfied are you with the vaccine you received today? & \multicolumn{2}{|c|}{$n=249$} & \multicolumn{2}{|c|}{$n=845$} & \multicolumn{2}{|c|}{$n=167$} \\
\hline Very satisfied & 167 & 67.1 & 404 & 47.8 & 121 & 72.5 \\
\hline Satisfied & 72 & 28.9 & 404 & 47.8 & 44 & 26.3 \\
\hline Somewhat satisfied & 9 & 3.6 & 22 & 2.6 & 2 & 1.2 \\
\hline Not satisfied & 1 & 0.4 & 15 & 1.8 & 0 & 0.0 \\
\hline What was the main reason for your satisfaction? & \multicolumn{2}{|c|}{$n=231$} & \multicolumn{2}{|c|}{$n=825$} & \multicolumn{2}{|c|}{$n=166$} \\
\hline The injection was minimally painful/only hurt a little & 137 & 59.3 & 468 & 56.7 & 118 & 71.1 \\
\hline I was reassured by the microneedle (short and thin needle) & 56 & 24.2 & 37 & 4.5 & 10 & 6.0 \\
\hline The vaccination/administration process was quick & 38 & 16.5 & 320 & 38.8 & 38 & 22.9 \\
\hline Other & 1 & 0.4 & 2 & 0.2 & 0 & 0.0 \\
\hline $\begin{array}{l}\text { For next year's flu, would you consider the following? } \\
\text { (day of vaccination) }\end{array}$ & \multicolumn{2}{|c|}{$n=245$} & \multicolumn{2}{|c|}{$n=844$} & \multicolumn{2}{|c|}{$n=167$} \\
\hline To be vaccinated with the same vaccine as today & 241 & 98.4 & 774 & 91.7 & 164 & 98.2 \\
\hline To be vaccinated with the intramuscular vaccine & 4 & 1.6 & 23 & 2.7 & 3 & 1.8 \\
\hline No vaccination & 0 & 0.0 & 47 & 5.6 & 0 & 0.0 \\
\hline $\begin{array}{l}\text { For next year's flu, would you consider the following? } \\
\text { ( } 8 \text { days after vaccination) }\end{array}$ & \multicolumn{2}{|c|}{$n=249$} & \multicolumn{2}{|c|}{$n=844$} & \multicolumn{2}{|c|}{$n=166$} \\
\hline To be vaccinated with the same vaccine as today & 233 & 93.6 & 723 & 85.7 & 161 & 97.0 \\
\hline To be vaccinated with the intramuscular vaccine & 13 & 5.2 & 57 & 6.8 & 4 & 2.4 \\
\hline No vaccination & 3 & 1.2 & 64 & 7.6 & 1 & 0.6 \\
\hline
\end{tabular}

$\mathrm{GP}=$ general practitioner.

Results were from a self-administered questionnaire. Percentages were calculated as $100 \times$ (number in each category

$[n] \div$ the number of responses available for each question $[n])$. 
and preference of i.d. versus i.m. vaccination were not significantly influenced by age, sex, or the feeling of being at risk for being infected with influenza (data not shown).

When asked immediately after vaccination, $98.4 \%$ of Turkish vaccinees, $98.2 \%$ of elderly Czech adults, and $91.7 \%$ of Czech adults 18-59 years of age indicated that they would like "to be vaccinated with the same vaccine as today" (Table 2). When asked again 8 days after vaccination, $93.6 \%$ of Turkish vaccinees, $85.7 \%$ of Czech adults 18-59 years of age, and $97.0 \%$ of Czech adults $\geq 60$ years of age indicated that they would like to receive the same vaccine next year. Overall, the 93.9\% preferred the same vaccine next year when asked immediately and $88.7 \%$ when asked again 8 days later.

\section{Prescriber Responses}

A total of 46 vaccine prescribers answered questionnaires, including 18 in Turkey and 28 in the Czech Republic. Most prescribers $(n=11)$ in Turkey were specialists, and all practiced in urban settings ( $>500$ inhabitants $/ \mathrm{km}^{2}$ ). In the Czech Republic, most vaccine prescribers $(n=24)$ were GPs, and $75 \%$ of prescribers practiced in urban settings, $11 \%$ in peri-urban settings (100-500 inhabitants $/ \mathrm{km}^{2}$ ), and $14 \%$ in rural areas $\left(<100\right.$ inhabitants $\left./ \mathrm{km}^{2}\right)$.

\section{Prescriber Satisfaction with Intanza/IDflu}

In Turkey, $100 \%$ of prescribers were satisfied or very satisfied with Intanza/IDflu, and all preferred i.d. over i.m. vaccine (Table 3 ). In the Czech Republic, 92.8\% were satisfied or very satisfied with Intanza/IDflu, and 71.4\% preferred i.d. over i.m. vaccine. Overall, 95.3\% were satisfied or very satisfied, and $82.6 \%$ preferred i.d. over i.m. vaccine.

\section{DISCUSSION}

This survey evaluated the acceptance in routine clinical practice of the first microneedle influenza vaccine, Intanza/ IDflu, by adult vaccinees and their vaccine prescribers during the 2010/2011 northern hemisphere influenza season. The study,

Table 3. Prescriber responses.

\begin{tabular}{|c|c|c|c|c|}
\hline & \multicolumn{2}{|c|}{ Turkey } & \multicolumn{2}{|c|}{ Czech Republic } \\
\hline & $n$ & $\%$ & $n$ & $\%$ \\
\hline How satisfied are you with the i.d. vaccine? & \multicolumn{2}{|c|}{$n=15$} & \multicolumn{2}{|c|}{$n=28$} \\
\hline Very satisfied & 5 & 33.3 & 17 & 60.7 \\
\hline Satisfied & 10 & 66.7 & 9 & 32.1 \\
\hline Somewhat satisfied & 0 & 0.0 & 2 & 7.1 \\
\hline Not satisfied & 0 & 0.0 & 0 & 0.0 \\
\hline Do you prefer i.d. over i.m. vaccine? & \multicolumn{2}{|c|}{$n=18$} & \multicolumn{2}{|c|}{$n=28$} \\
\hline Yes & 18 & 100.0 & 20 & 71.4 \\
\hline No & 0 & 0.0 & 8 & 28.6 \\
\hline
\end{tabular}

i.d.=intradermal; i.m.=intramuscular.

Results were from a self-administered questionnaire. Percentages were calculated as $100 \times$ (number in each category $[n] \div$ the number of responses available for each question $[n])$. 
which included 1261 vaccinees and 46 vaccine prescribers in the Czech Republic and Turkey, showed a high rate of acceptance of i.d. vaccination with Intanza/IDflu by both vaccinees and vaccine prescribers.

Overall, 96.1\% of vaccinees reported being satisfied or very satisfied with Intanza/ IDflu, with similar rates in Turkey and the Czech Republic, and irrespective of age. Also, overall, 93.9\% said they would prefer the same vaccination (i.d.) when asked immediately after vaccination, and $88.7 \%$ responded that they would prefer the same vaccine when asked again 8 days later. This suggests that any reactivity during the week following the vaccination had little effect on vaccine acceptability. In addition, more than $90 \%$ of those vaccinated every year were satisfied or very satisfied with Intanza/IDflu, indicating that satisfaction rates for Intanza/IDflu were high even in subjects previously having received i.m. vaccination.

Similar results were found in a 2010 survey of adult vaccinees 18-59 years of age in Australia and Argentina, with 98\% reporting being satisfied or very satisfied with Intanza/ IDflu $9 \mu \mathrm{g} .{ }^{26}$ Similarly, a survey of vaccinees in two phase 3 studies of Intanza/IDflu found that $96 \%$ of vaccinees were satisfied with the injection, and more than $96 \%$ considered the injection to be very or totally acceptable. ${ }^{27}$ Also, similar to the current survey, $87 \%$ of vaccinees in Argentina reported a preference for receiving the same injection the following year when asked immediately after the vaccination, and $86 \%$ when asked again after 7-10 days. ${ }^{26}$ Collectively, the previous and current surveys show that vaccinee satisfaction is high for Intanza/IDflu, regardless of the country or age group.

In both Turkey and the Czech Republic, minimal pain of injection was the main reason for satisfaction reported by vaccinees. This was the same main reason for satisfaction in the Australia/Argentina survey. ${ }^{26}$ In contrast, a recent survey of the general public in France and Germany following an online presentation of Intanza/IDflu (and in the absence of vaccination) found that the thin, short needle is perceived as the most important benefit, and less pain or pain-free administration is the fourth most important benefit. ${ }^{28}$ Being reassured by the short and thin microneedle was the second (Turkey) or third most (Czech Republic) common reason for satisfaction in the current survey. Clearly, there are differences in the perceived benefits of Intanza/IDflu according to whether or not those participating in the surveys had received the injection, and different reasons motivating individuals to select i.d. vaccination with Intanza/ IDflu for the first time and subsequent times.

Professional opinion in favor of i.d. influenza vaccination with Intanza/IDflu was high, according to this survey. In Turkey, all prescribers indicated that they were satisfied or very satisfied with Intanza/IDflu, and all preferred i.d. over i.m. vaccination. In the Czech Republic, more than $90 \%$ were satisfied or very satisfied with Intanza/IDflu, and approximately threequarters preferred i.d. over i.m. vaccination. These results agree well with those from the previous Australia/Argentina survey, where 85\% were satisfied or very satisfied with Intanza/IDflu and $74 \%$ preferred i.d. over i.m. vaccination. ${ }^{26}$

Although satisfaction with Intanza/ IDflu was high in both Turkey and the Czech Republic, the attitudes about seasonal influenza differed between the countries and between age groups. Only approximately $10 \%$ of vaccinees in Turkey (most of whom were 18-59 years old) and vaccinees 60 years or older in the Czech Republic felt that they were not at risk of catching the flu. In contrast, approximately $33 \%$ of the 18-59-year age group in the Czech Republic 
did not feel at risk. Although this suggests that feelings of risk of catching the flu might be similar in elderly adults in the Czech Republic and the vaccinees in Turkey, reasons for feeling or not feeling at risk differed. For example, coming into contact with many people was the most common reason for feeling at risk in Turkey and in vaccinees 18 -59 years of age in the Czech Republic, whereas having a chronic illness was the most common reason in elderly Czech adults. Furthermore, relying on natural defenses was the most common reason for not feeling at risk in Turkey and in vaccinees 18-59 years of age in the Czech Republic, whereas not having a chronic illness was the most common reason in elderly Czech adults. In other words, for elderly Czech adults, whether they had a chronic illness was the main factor influencing whether they were vaccinated for influenza, whereas other factors were more important for younger Czech adults and for the vaccinees in Turkey.

In the Czech Republic, approximately twothirds of vaccinees 18-59 years old and 80\% of elderly respondents reported receiving the influenza vaccination every year, and more than $80 \%$ in both young adults and elderly respondents reported having been vaccinated for influenza the previous year. These coverage rates are much higher than in Turkey, where only $27.0 \%$ reported being vaccinated every year and only $57.1 \%$ reported being vaccinated the year before. For the vaccinees in the Czech Republic that missed recent vaccinations, the most common reason was that they did not feel at risk of catching the flu, and in Turkey, the most common reason for missing recent vaccinations was not being encouraged to be vaccinated. Importantly, for all vaccinees, advice of the physician or a GP was considered the most effective reminder to receive the influenza vaccination. A postcard, email, or text message sent by the physician's office or clinic was considered the second most effective reminder. This agrees with the conclusions of the previous survey in Argentina and Australia. ${ }^{26}$ Therefore, educational efforts should probably focus on encouraging physicians to discuss seasonal influenza vaccination with their patients and to remind them to be vaccinated before the influenza season.

As in the Australia/Argentina survey, ${ }^{26}$ the results of this survey need to be interpreted in light of specific aspects of the study design and vaccinee profile. In particular, only subjects electing to receive i.d. vaccination with Intanza/IDflu were included in the survey, so the authors could not directly compare the acceptability of i.m. and i.d. influenza vaccination. Including only those electing to receive i.d. vaccination might also have biased the results in favor of i.d. vaccination. However, the results were nearly the same as determined in phase 3 clinical trials that also assessed satisfaction with Intanza/IDflu. ${ }^{27}$ Also, although the authors assessed vaccinee satisfaction immediately and 8 days after vaccination, reactivity was not assessed, so the impact of reactivity on satisfaction could not be directly determined. Finally, 14 of the 249 vaccinees in Turkey were $\geq 60$ years of age and were vaccinated with Intanza/IDflu $9 \mu \mathrm{g}$. However, these vaccinees represent only $6.4 \%$ of the total in the Turkish survey, so this should not substantially affect the conclusions.

In conclusion, collectively, these data and the authors' previous results ${ }^{26}$ show that Intanza/ IDflu is well accepted by vaccinees and vaccine prescribers in routine clinical practice. Intanza/ IDflu might have the additional benefit of increasing vaccination rates in adults against seasonal influenza by offering an alternative vaccine with a smaller needle, as well as a minimally painful injection. Future studies are needed to assess the impact of Intanza/IDflu on influenza vaccination rates. 


\section{ACKNOWLEDGMENTS}

This study was funded by Sanofi Pasteur. Medical writing assistance in the preparation of this manuscript was provided by Dr. Phillip Leventhal (4Clinics, Paris, France). Financial support for this assistance was provided by Sanofi Pasteur. Potential conflicts of interest are as follows: F.W., S.A., and R.S. are employees of Sanofi Pasteur; R.P. and G.U. have served as advisors for government, industry, and academic research organizations. They have received financial compensation or expense reimbursement from Sanofi Pasteur in relation to presentations and participation in advisory panels. F.W. is the guarantor for this article and takes responsibility for the integrity of the work as a whole.

Open Access. This article is distributed under the terms of the Creative Commons Attribution Noncommercial License which permits any noncommercial use, distribution, and reproduction in any medium, provided the original author(s) and source are credited.

\section{REFERENCES}

1. Monto AS. Seasonal influenza and vaccination coverage. Vaccine. 2010;28(suppl. 4):D33-D44.

2. World Health Organization. Influenza (seasonal). Available at: http://www.who.int/mediacentre/ factsheets/fs211/en/index.html. Accessed October 6, 2011.

3. Thompson WW, Shay DK, Weintraub E, et al. Mortality associated with influenza and respiratory syncytial virus in the United States. JAMA. 2003;289:179-186.

4. Glezen WP, Greenberg SB, Atmar RL, et al. Impact of respiratory virus infections on persons with chronic underlying conditions. JAMA. 2000;283:499-505.

5. Barker WH. Excess pneumonia and influenza associated hospitalization during influenza epidemics in the United States, 1970-78. Am J Public Health. 1986;76:761-765.
6. Barker WH, Mullooly JP. Impact of epidemic type A influenza in a defined adult population. Am J Epidemiol. 1980;112:798-811.

7. Molinari NA, Ortega-Sanchez IR, Messonnier ML, et al. The annual impact of seasonal influenza in the US: measuring disease burden and costs. Vaccine. 2007;25:5086-5096.

8. Xue Y, Kristiansen IS, de Blasio BF. Modeling the cost of influenza: the impact of missing costs of unreported complications and sick leave. BMC Public Health. 2010;10:724.

9. Nichol KL, Lind A, Margolis KL, et al. The effectiveness of vaccination against influenza in healthy, working adults. $\mathrm{N}$ Engl J Med. 1995;333:889-893.

10. Bridges CB, Thompson WW, Meltzer MI, et al. Effectiveness and cost-benefit of influenza vaccination of healthy working adults: a randomized controlled trial. JAMA. 2000;284:1655-1663.

11. World Health Organization. Resolution of the World Health Assembly WHA 56.19. Prevention and control of influenza pandemics and annual epidemics. 56th World Health Assembly, Geneva 2003.

12. The Council of the European Union. Council recommendation of 22 December 2009 on seasonal influenza vaccination. Official Journal of the European Union. 2009;L348:71-72.

13. Fiore AE, Uyeki TM, Broder $\mathrm{K}$, et al. Prevention and control of influenza with vaccines: recommendations of the Advisory Committee on Immunization Practices (ACIP), 2010. MMWR Recomm Rep. 2010;59:1-62.

14. Centers for Disease Control. Healthy People 2020 Summary of Objectives. Available at: http:// healthypeople.gov/2020/topicsobjectives2020/ pdfs/Immunization.pdf. Accessed March 7, 2011.

15. Centers for Disease Control and Prevention. Interim results: state-specific seasonal influenza vaccination coverage - United States, August 2009-January 2010. MMWR Morb Mortal Wkly Rep. 2010;59:477-484.

16. Prausnitz MR, Mikszta JA, Cormier $\mathrm{M}$, et al. Microneedle-based vaccines. Curr Top Microbiol Immunol. 2009;333:369-393.

17. Laurent PE, Bonnet S, Alchas P, et al. Evaluation of the clinical performance of a new intradermal vaccine administration technique and associated delivery system. Vaccine. 2007;25:8833-8842. 
18. European Medicines Agency. Assessment report for Intanza ${ }^{\circledR}$. Available at: http://www.ema. europa.eu/ema/index.jsp?curl=pages/medicines/ human/medicines/000957/human_med_000842. jsp\&murl=menus $/$ medicines $/$ medicines.jsp\&mid= WC0b01ac058001d125\&jsenabled=true. Accessed December 2, 2011.

19. Arnou R, Eavis P, Pardo JR, et al. Immunogenicity, large scale safety and lot consistency of an intradermal influenza vaccine in adults aged 18-60 years: Randomized, controlled, phase III trial. Hum Vaccin. 2010;6:346-354.

20. Arnou R, Icardi G, De Decker M, et al. Intradermal influenza vaccine for older adults: a randomized controlled multicenter phase III study. Vaccine. 2009;27:7304-7312.

21. Beran J, Ambrozaitis A, Laiskonis A, et al. Intradermal influenza vaccination of healthy adults using a new microinjection system: a 3-year randomised controlled safety and immunogenicity trial. BMC Med. 2009;7:13.

22. Holland D, Booy R, De Looze F, et al. Intradermal influenza vaccine administered using a new microinjection system produces superior immunogenicity in elderly adults: a randomized controlled trial. J Infect Dis. 2008;198:650-658.
23. Leroux-Roels I, Vets E, Freese R, et al. Seasonal influenza vaccine delivered by intradermal microinjection: a randomised controlled safety and immunogenicity trial in adults. Vaccine. 2008;26:6614-6619.

24. Güvenlik Kurumu Saslık Uygulama Talimatı. Directive for Practice of Health. Available at: http://www.sgk.gov.tr/wps/portal/ESGK/GSS. Accessed December 2, 2011.

25. Blank PR, Szucs TD. Increasing influenza vaccination coverage in recommended population groups in Europe. Expert Rev Vaccines. 2009;8:425-433.

26. Eizenberg P, Booy R, Naser N, et al. Acceptance of Intanza ${ }^{\circledR} 9 \mu \mathrm{g}$ intradermal influenza vaccine in routine clinical practice in Australia and Argentina. Adv Ther. 2011;28:640-649.

27. Reygrobellet C, Viala-Danten M, Meunier J, et al. Perception and acceptance of intradermal influenza vaccination: patient reported outcomes from phase 3 clinical trials. Hum Vaccin. 2010;6:336-345.

28. Arnou R, Frank M, Hagel T, et al. Willingness to vaccinate or get vaccinated with an intradermal seasonal influenza vaccine: a survey of general practitioners and the general public in France and Germany. Adv Ther. 2011;28:555-565. 